\title{
Usefulness of assessing the descending aorta flow pattern for predicting the need for intervention in patients with aortic coarctation.
}

\author{
Begoña Igual Muñoz ${ }^{*}$, Diana Domingo-Valero ${ }^{2}$, Alicia Maceira-Gonzalez ${ }^{3}$, Joaquin Rueda-Soriano ${ }^{2}$, \\ Francisco Valera $^{2}$, Jose Ignacio Carrasco ${ }^{2}$, Alejandro Sanchez-Vazquez ${ }^{3}$, Anastasio Montero-Argudo ${ }^{3}$ \\ ${ }^{1}$ Department of Cardiology, Consorcio Hospital General Universitario de Valencia, Spain \\ ${ }^{2}$ Department of Cardiology, Hospital Universitari i Politecnic La Fe, Spain \\ ${ }^{3}$ Centro Medico ERESA, Spain
}

\begin{abstract}
Background: Nowadays, evaluation of the need for intervention in patients with aortic coarctation (AC) is based on anatomical and clinical data. The purpose of the study was to analyze the utility of assessing flow in the thoracic descending aorta using magnetic resonance imaging (MRI) in patients with AC.

Methods: Patients with a diagnosis or suspicion of AC, who underwent MRI study between 2008-2016 in a single tertiary center, were retrospectively studied. MRI standardized protocol included gadolinium enhanced angiographic 3-D sequences to evaluate relative stenosis (RS) and phase contrast imaging to assess flow in the descending aorta.

Results: 106 patients $\{70$ men $(66 \%)$, mean age $22 \pm 17$ years $\}$ were included. Of these, $28(26 \%)$ required surgical intervention during the first year after evaluation and $9(9.3 \%)$ required percutaneous treatment. AUROC curves were applied to find best cut-off points of imaging variables. Imaging variables were entered into a multivariate logistic regression model including the presence of arterial hypertension. Percentage of flow increase was shown to be an independent predictor of the need for an intervention (OR, 7.2; 95\% CI, 1-20; $p=0.03)$ and adding this parameter in the standard work-up represented a significant increase in diagnostic accuracy $(\mathrm{S}, 85 \% ; \mathrm{Sp}, 89 \%$; vs. S, 62\%; Sp, 92\%).

Conclusion: physiological assessment of flow pattern in descending aorta by MRI significantly increases the ability to evaluate the need for invasive treatment in $\mathrm{AC}$ patients beyond standard clinical and anatomical study.
\end{abstract}

Keywords: Aortic coarctation, Magnetic resonance, Treatment.

Accepted on June 04, 2018

\section{Introduction}

Aortic coarctation (AC) accounts for 5\%-8\% of all congenital heart diseases and affects 3 of every 10,000 live born infants [1]. Despite being considered a diffuse vascular condition, AC mainly takes the form of stenosis in the descending thoracic aorta distal to the origin of the left subclavian artery.

The current recommendations of the European Society of Cardiology for invasive treatment of $\mathrm{CoA}$ are based on clinical and imaging data [2], thus an image-proven relative stenosis greater than $50 \%$ was considered as a class II recommendation. Nevertheless, assessment of the anatomical severity of stenosis is not free of limitations, for this reason a pressure gradient $>20$ $\mathrm{mmHg}$ measured in a hemodynamics laboratory remains as the gold standard for evaluation.

However, lower gradients have also been associated with persistent arterial hypertension [3] thus indicating that invasive treatment could be indicated in less severe stenosis if other clinical or imaging data suggest physiological repercussion of the stenosis. These reasons illustrate the complexity of this evaluation that requires all available information to improve selection of patients who would benefit from invasive procedures.

Recent studies have reported good association between MRI variables and the hemodynamic severity of AC obtained in catheterization laboratory [4-8]. The objective of these studies was to validate MRI as an ideal noninvasive method for the evaluation of patients with AC. The present study carries forward these efforts to evaluate the clinical usefulness of MRI for assessing the need for invasive treatment in a cohort of patients with AC.

\section{Methods}

An observational retrospective cohort study including patients with a diagnosis or suspicion of $\mathrm{AC}$ who underwent clinical and MRI evaluation in a single tertiary center between January 2008 and September 2016 were included. Written consents were obtained before MRI evaluation and confidentiality of the data was guaranteed. Clinical assessment was based on the electronic clinical reports available on local software package. 
Primary outcome was the indication of invasive treatment within the first year after evaluation and this issue was left to the discretion of clinical cardiologist and performed according to standard clinical practice and guidelines of European Society of Cardiology [2].

\section{Clinical assessment of aortic coarctation}

Clinical assessment of arterial hypertension was carried out by a team of physicians with expertise in congenital heart disease evaluation. Arterial hypertension was reported in 47 patients (44\%) and a pressure gradient between the lower and upper limbs was also reported in 15 patients of the group.

\section{Cardiac magnetic resonance imaging}

The MRI studies were made with a 1.5-T system (Syngo HDX GE® Milwaukee, USA) with available cardiac software.
The CMR protocol included the following: Phase- contrast sequences with through-plane flow encoding were prescribed in planes centered in the descending aorta and aligned orthogonally to the expected main blood flow direction. Usual imaging parameters were slice thickness $5-8 \mathrm{~mm}$; in-plane resolution $256 \times 128 \mathrm{~mm}$. Temporal resolution of at least 20 frames by heartbeat was obtained with retrospective EKG trigger and segmented k-space reconstruction within limits of breath holding capabilities. Velocity encoding sensitivity (Venc) was adapted to the vessel velocity and after each scan, phase difference images were checked for aliasing. If aliasing is present, Venc was adapted accordingly. Phase-contrast sequences were acquired in two slices, one immediately proximal to the coarctation site (slice A) and the second was acquired in the descending aorta at the level of the diaphragm (slice B) (Figure 1).
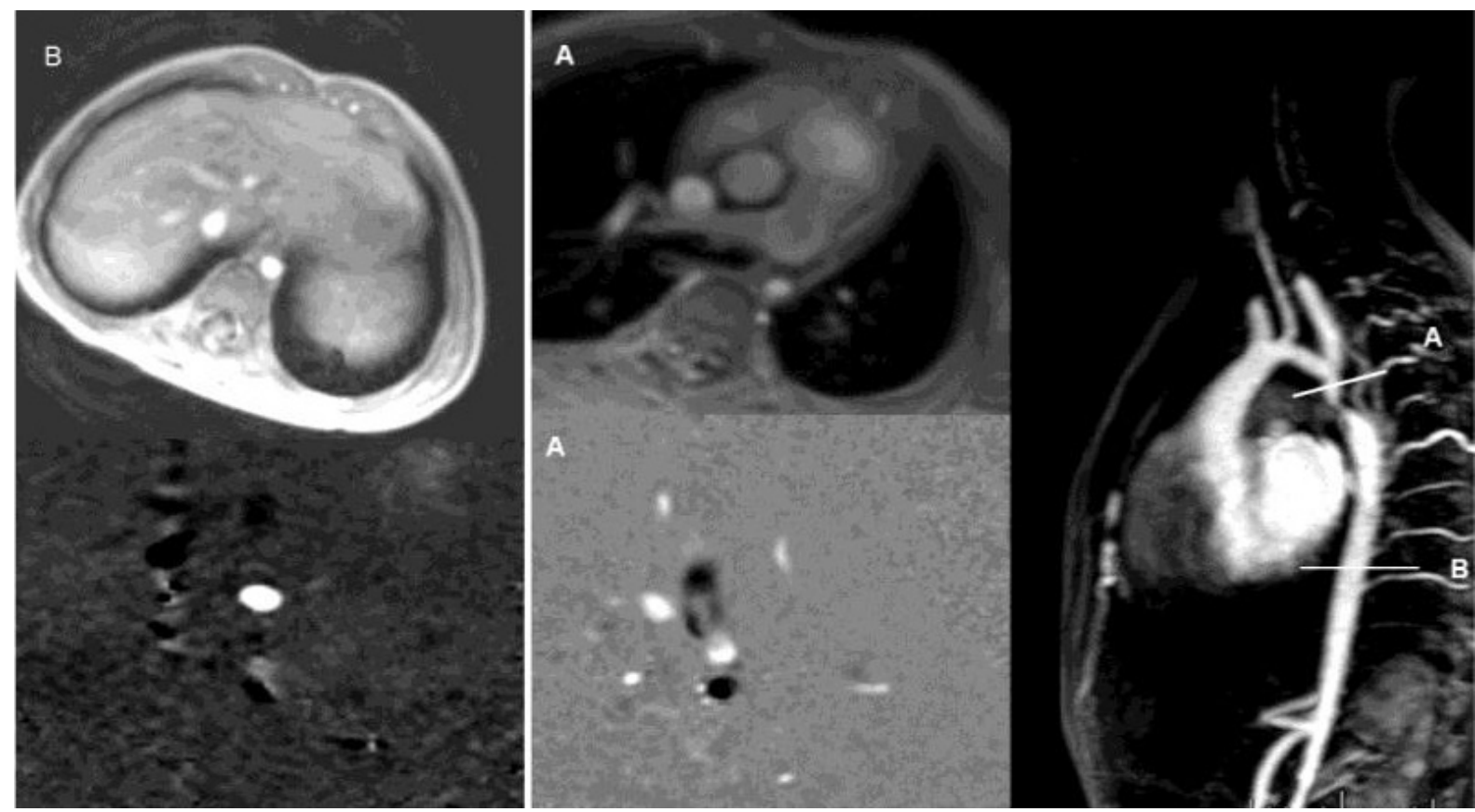

Figure 1. CMR phase-contrast sequences for the evaluation of physiological parameters in coarctation of the aorta. Slice A, proximal to the stenosis site; slice B, in the descending aorta at the level of the diaphragm.

Angiographic sequences: 128-134 slices with an spatial resolution of $0.8-1.5$ were obtained using breath hold fast gradient echo 3D after an intravenous bolus of $0.2 \mathrm{ml} / \mathrm{kg}$ of gadolinium chelate (Gadobenate dimeglumine, Multihance ${ }^{\circledR}$ Bracco imaging, Italia) administered at $5 \mathrm{ml} / \mathrm{s}$ and tracking the bolus for the location of the contrast in the thoracic aorta.

\section{Post processing}

The phase contrast sequences were analyzed using Report CARD ${ }^{\circledR}$ (General Electric, Milwaukee, USA) by evaluating the total flow volume immediately proximal to the CoA in slice $A$ and the total flow volume in the descending aorta at the level of the diaphragm in slice B. Figure 1 shows the acquisition methodology for the variables mentioned. Multiplane reconstructions of the $3 \mathrm{D}$ angiographic sequences were performed (Advantage Windows 4.5 workstation, GE®, Milwaukee, USA) to obtain the minimum diameter at the CoA site together with the diameter in the descending aorta at the level of the diaphragm (Figure 2). These data were used, as described elsewhere [9], to obtain the relative stenosis, which was defined as follows: \{maximum diameter of the descending aorta at the level of the diaphragm - minimum diameter in the CoA site $\} /$ maximum diameter of the descending aorta $\mathrm{x} 100$. Percentage of flow increase at descending aorta was defined as follows: \{flow in the descending aorta at the level of the diaphragm - flow in the descending aorta proximal to the $\mathrm{CoA}\}$ /flow in the proximal aorta $\mathrm{x} 100$. 
Citation: Igual Muñoz B, Domingo-Valero D, Maceira-Gonzalez A, et al. Usefulness of assessing the descending aorta flow pattern for predicting the need for intervention in patients with aortic coarctation. Ann Cardiovasc Thorac Surg 2018;1(1):24-29.

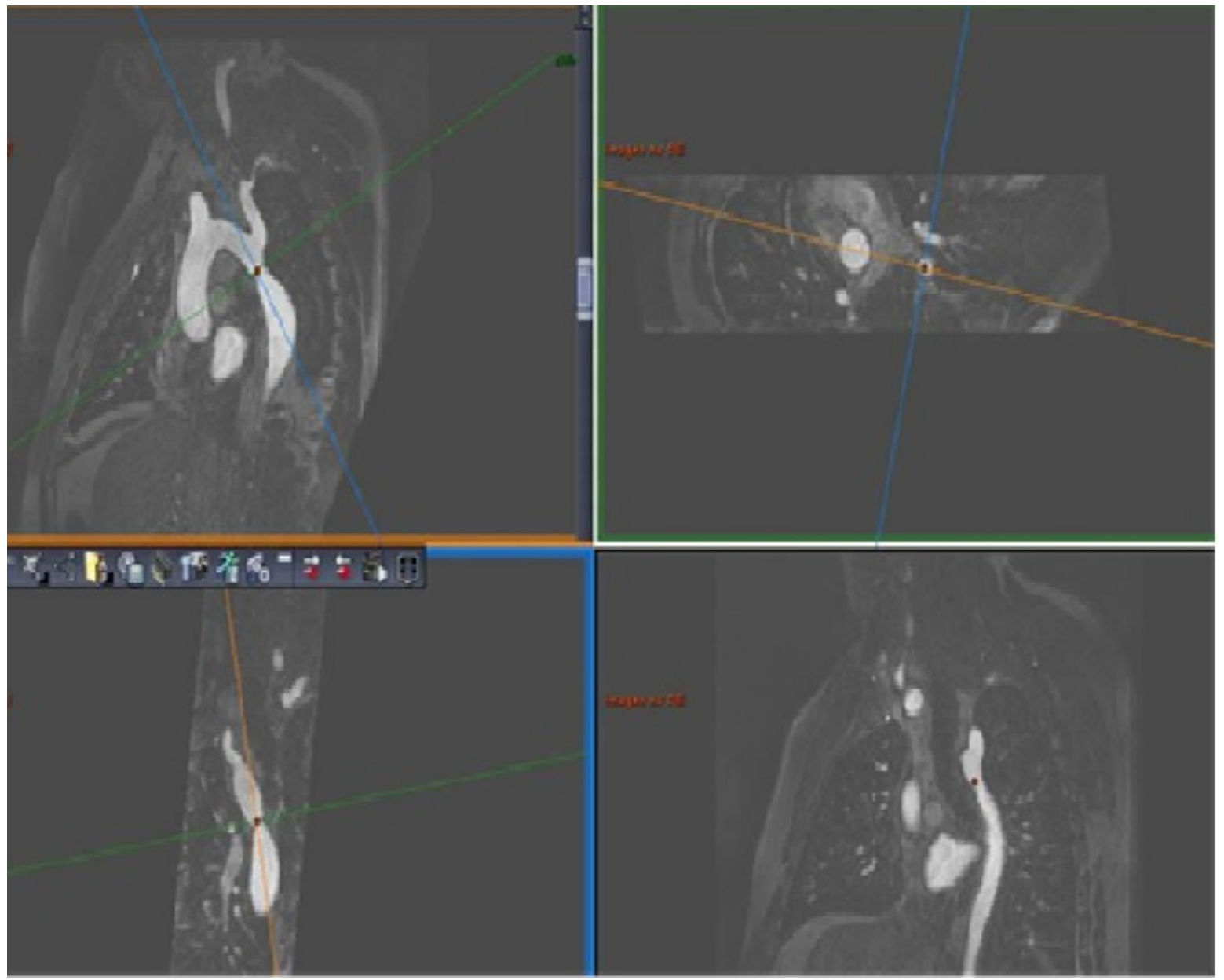

Figure 2. CMR post processing of 3-D angiographic sequences to quantify relative stenosis using multiplane reconstructions in order to obtain in a double oblique plane transversal to the aorta wall the minimum diameter at the coarctation site and maximum diameter in the aorta at the level of the diaphragm.

Both relative stenosis and percentage of flow increase were entered into the binary logistic regression model as dichotomous variables. The best cut-off point obtained after analyzing the area under the receiver operating characteristic (ROC) curve was considered pathological. An increased flow $\geq$ $15 \%$ and relative stenosis $\geq 48 \%$ were shown to be the best cut-off points, sensitivity and specificity of analyzed variables were shown in the table 2.

\section{Statistical analysis}

Qualitative variables are expressed as percentages and quantitative variables as the mean \pm 2 standard deviations. The Kolmogorov-Smirnov test was used to evaluate whether the variables were distributed normally. Qualitative variables were analyzed using the chi-square test with a Yates correction or the Fisher exact text when the expected frequencies were less than 3 and 5, respectively. The best cut-off for dichotomous analysis for relative stenosis and flow increase was obtained by analyzing the area under the ROC curve.

A multivariate analysis was performed using a binary logistic regression model including arterial hypertension, dichotomized relative stenosis and dichotomized flow increase. The dependent variable was the need for an intervention during the first year after cardiac magnetic resonance. The diagnostic accuracy of the models was evaluated by analyzing the sensitivity and specificity of the predictive model. Analysis of the reproducibility of the CMR data was based on inter and intraobserver variability. The analysis was performed using SPSS 15.0 (Chicago, Illinois, USA). Statistical significance was set at $\mathrm{P}>0.05$.

\section{Results}

The study population comprised 120 patients, of whom 14 were excluded ( 2 with metallic artifacts at the study site, 12 who were lost to follow-up or had incomplete clinical or CMR data). The final sample comprised 106 patients $\{70$ males $(66 \%)$, mean age $22 \pm 17$ years $\}$. Of these, 28 (26\%) required and intervention during the first year 9 of them (8.5\%) required percutaneous treatment. Demographic data are shown in Table 1.

\section{CRM parameters associated with the need for an intervention to treat $A C$}

The area under the ROC curve was used to analyze the diagnostic value of the imaging variables with respect to need for an intervention to treat AC. The area under the curve, 
confidence interval, statistical significance, and optimal cut-off point, together with the sensitivity and specificity for each of the parameters are shown in Table 2.

Table 1. Demographic data.

\begin{tabular}{llll}
\hline $\mathbf{N}=106$ Patients & Intervention & No intervention & $\mathbf{p}$ \\
\hline Age & $18 \pm 16$ & $24 \pm 16$ & $\mathrm{NS}$ \\
\hline Males, \% & $78 \%$ & $60 \%$ & $\mathrm{NS}$ \\
\hline Body area & $1.16 \pm 0.6$ & $1.62 \pm 0.3$ & $\mathrm{P}<0.05^{*}$ \\
\hline Relative stenosis & $66 \pm 23 \%$ & $34 \pm 17 \%$ & $\mathrm{P}<0.001^{\text {** }}$ \\
\hline Increased flow & $49 \pm 26$ & $7 \pm 19$ & $\mathrm{P}<0.001^{\text {** }}$ \\
\hline LVEDVi & $58 \pm 37 \mathrm{ml} / \mathrm{m}^{2}$ & $68 \pm 39 \mathrm{ml} / \mathrm{m}^{2}$ & $\mathrm{NS}$ \\
\hline RVEDVi & $36 \pm 33$ & $49 \pm 33$ & $\mathrm{NS}$ \\
\hline LV ejection fraction & $68 \pm 9 \%$ & $66 \pm 10 \%$ & $\mathrm{NS}$ \\
\hline RV ejection fraction & $57 \pm 8 \%$ & $59 \pm 11 \%$ & $\mathrm{NS}$ \\
\hline Previous therapy & $4(8.5 \%)$ & $43(91.5 \%)$ & $\mathrm{P}<0.001^{\text {** }}$ \\
\hline
\end{tabular}

LV: left ventricle; RD: right ventricle; LVEDVi: left ventricular end-diastolic volume index; RVEDVi: right ventricular end-diastolic volume index; * $p<0.05 ;{ }^{* *} p<0.001$.

Dichotomous RS and percentage of flow increase in descending aorta and also presence of arterial hypertension at evaluation were entered in a multivariate binary logistic regression model. Table 3 shows the statistical significance and odds ratios obtained.

Table 2. Diagnostic performance, area under ROC curve, statistical significance and cut-off points of imaging variables.

\begin{tabular}{llllll}
\hline Variables & $\begin{array}{l}\text { ROC } \\
\text { curve }\end{array}$ & P & CI & $\begin{array}{l}\text { Cut-off } \\
\text { point }\end{array}$ & S/Sp \% \\
\hline Increased flow, \% & 0.9 & $<0.001$ & $0.82-0.98$ & $15 \%$ & $88 / 99$ \\
\hline Relative stenosis & 0.85 & $<0.001$ & $0.75-0.95$ & $48 \%$ & $82 / 79$ \\
\hline
\end{tabular}

ROC: receiver operating characteristic; $\mathrm{Cl}$, confidence interval; S, sensitivity; Sp, specificity.

Percentage of flow increase was an independent predictor of the need for an intervention (OR, 7.2; $\mathrm{p}=0.03 ; \mathrm{CI}, 1-20)$ and adding this parameter in the standard work-up led to a significant increase in diagnostic accuracy (S, 85\%; Sp, 89\%; vs. S, $62 \%$; Sp: 92\%). Table 3 shows the statistical significance and odds ratios obtained.

\section{Reproducibility of imaging variables studied using CRM}

The intra- and inter-observer variability of flow volume measurements at thoracic descending aorta was assessed in a subset of 33 patients with a variability of $6 \%$.
Table 3. Stepwise multivariable binary logistic regression analysis.

\begin{tabular}{|c|c|c|c|}
\hline Multivariate analysis. MODEL A & B & Sig. & $\operatorname{Exp}(B)$ \\
\hline Relative stenosis ${ }^{a}$ & 0.017 & 0 & 10.018 \\
\hline Arterial hypertension & 2.529 & 0.008 & 5.14 \\
\hline Constant & -4.52 & 0 & 0.042 \\
\hline Multivariate analysis. MODEL B & B & Sig. & $\operatorname{Exp}(B)$ \\
\hline Relative stenosis ${ }^{a}$ & 0.017 & 0.33 & 4.81 \\
\hline Arterial hypertension & 2.529 & 0.03 & 4.63 \\
\hline Increased flow ${ }^{b}$ & 4.435 & 0.002 & 11.02 \\
\hline Constant & -3.9 & 0 & 0.19 \\
\hline
\end{tabular}

a $\geq 48 \%$ at the site of CoA referenced to the descending aorta at the level of the diaphragm,

$b \geq 15 \%$ in the distal descending aorta.

\section{Discussion}

New imaging technologies have been developing considerably and sophisticated post processing tools for analysis of vessel anatomy are now available. In addition, they make it possible to assess new physiological parameters, such as flow pattern in the descending aorta, which enables noninvasive assessment of the hemodynamic consequences of stenosis at the coarctation site.

In this clinical scenario, both anatomical and physiological parameters have proven useful for predicting the need for intervention to treat AC. Muzzarelli et al. [10] showed that a logistic regression model combining the minimum aortic crosssectional area indexed to body surface area, together with the rate-corrected deceleration time, provided the best predictive model in their study. Our findings are consistent with those reported above, since we also observed that the best diagnostic model included both anatomical and physiological parameters. However, the fact that we analyzed different parameters means that our results are also different.

Our data show that not only relative stenosis at the coarctation site referenced to the diaphragmatic aorta but also the presence of a pathological flow pattern in the descending aorta were independent predictors of the need for intervention.

This finding is explained by the fact that the presence of significant stenosis leads to a fall in pressure in the vessel distal to the stenosis and to an inversion of the flow direction in the small arterial branches of the descending aorta, e.g., the bronchial and intercostal arteries. Therefore, in a healthy patient, flow volume in the descending aorta decreases slightly from the proximal portion to the distal portion of the descending aorta owing to presence of small arterial branches and the opposite occurs in patients with $\mathrm{AC}$, because the inversion of the flow direction in these small arterial branches . Therefore, the presence of a pathological flow pattern shows that the drop in pressure in the descending aorta causes inversion of the flow in small arterial branches and therefore 
Citation: Igual Muñoz B, Domingo-Valero D, Maceira-Gonzalez A, et al. Usefulness of assessing the descending aorta flow pattern for predicting the need for intervention in patients with aortic coarctation. Ann Cardiovasc Thorac Surg 2018;1(1):24-29.

stenosis at AC site is hemodynamically significant. This parameter has also been studied by Muzzarelli et al. [10]; however, the authors found aortic flow deceleration time to be the most powerful predictor.

These findings may be associated with the method of acquisition, since, although increased flow velocity is a classic variable [10], its acquisition and interpretation continue to be subject to methodological variability and its application in clinical practice is not fully established.

As for methodology, the Society for Cardiovascular Magnetic Resonance [11] recommends acquisition of flow sequences in the area immediately proximal or immediately distal to the coarctation and in the descending aorta at the level of the diaphragm. These recommendations could lead to differences in results. It must be taken into account that in order to quantify vessel flow volume, placement of the imaging plane in regions of turbulent flow can lead to signal loss and inaccuracies and should be avoided. According to this, the optimal site for evaluating flow pattern in the descending aorta might be proximal to stenosis site. In contrast, evaluation of the flow pattern in a slice immediately distal to the stenosis could provide a more accurate picture of stenosis-related parameters such as the peak gradient or the flow deceleration time, which have proven to be useful in other imaging series $[13,14]$. These methodological differences could account for the different findings and perhaps also be linked to the delay in these variables becoming part of clinical practice.

This is an observational study and it must be taken into account this possible source of information bias. Nevertheless the use of this physiological approaching using CMR imaging might prove useful as a part of diagnostic evaluation in AC patients and warrants further investigations.

This work consolidates the clinical usefulness of imaging techniques in the evaluation of patients with $\mathrm{CoA}$, in particular, with respect to MRI, which enables simultaneous study of anatomical and physiological parameters without the need for ionizing radiation.

Our findings emphasize the clinical impact of 2 easily acquired and highly reproducible imaging variables that can be analyzed quickly and are currently available in MRI units. We therefore aim to encourage and establish the use of this technology by recommending its routine incorporation into daily clinical practice.

However, it is important to consider that the present study was limited by its retrospective and observational nature; and must be taken into account as a possible source of selection and information bias.

\section{Limitation}

The present study was limited by its single center and its retrospective and observational nature; and it must be taken into account this possible source of selection and information bias.

\section{Conclusion}

MRI-based evaluation of the flow pattern in the descending aorta makes it possible to analyze the physiological consequences of AC. Therefore, adding the percentage of flow increase in descending aorta to the standard diagnosis significantly increases the ability to predict the need for an intervention in patients with $\mathrm{AC}$.

\section{Key points}

Cardiac magnetic resonance is a noninvasive technique that enables integral evaluation of anatomical and physiological parameters. Previous studies showed that comprehensive assessment of anatomical and physiological parameters increases diagnostic performance in this clinical setting.

A multivariate model including relative stenosis and the presence of arterial hypertension together with percentage of flow increase in the descending aorta showed that this physiological parameter is an independent predictor of the need for invasive treatment within first year after evaluation. Therefore this parameter significantly increases the ability to predict the need for intervention in patients with $\mathrm{AC}$ beyond clinical and anatomical evaluation.

\section{References}

1. SEC Working Group for ESC 2014 Guidelines on Diagnosis and Treatment of Aortic Diseases, Expert Reviewers for ESC 2014 Guidelines on Diagnosis and Treatment of Aortic Diseases, SEC Clinical Practice Guidelines Committee. Comments on the 2014 ESC guidelines on the diagnosis and treatment of aortic diseases. Rev Esp Cardiol (Engl Ed). 2015;68(3):179-84.

2. Erbel R, Aboyans V, Boileau C, et al. Corrigendum to: 2014 ESC Guidelines on the diagnosis and treatment of aortic diseases. Eur Heart J. 2015;36(41):2779.

3. Muzzarelli S, Ordovas KG, Hope MD, et al. Diagnostic value of the flow profile in the distal descending aorta by phase-contrast magnetic resonance for predicting severe coarctation of the aorta. J Magn Reson Imaging. 2011; 33(6):1440-6.

4. Bolzhauser M, Braun N, Alscher MD, et al. Hypertension due to aortic coarctation - a missed clinical diagnosis. Dtsch Med Wochenschr. Mayo de 2011; 136(19):1003-6.5.

5. Nielsen JC, Powell AJ, Garneau K, et al. Magnetic resonance imaging predictors of coarctation severity. Circulation. 2005;111(5):622-8.

6. Binnetoglu K, Yildiz CE, Babaoglu K, et al. Management of an ascending aortic aneurysm associated with a bicuspid aortic valve and a coarctation in an adolescent. $\mathrm{J}$ Card Surg. 2011;26(6):635-7.

7. Goubergrits L, Riesenkampff E, Yevtushenko P, et al. MRI-based computational fluid dynamics for diagnosis and treatment prediction: clinical validation study in patients with coarctation of aorta. J Magn Reson Imaging.

8. 2015;41(4):909-16. 
9. Muzzarelli S, Meadows AK, Ordovas KG, et al. Prediction of hemodynamic severity of coarctation by magnetic resonance imaging. Am J Cardiol. 2011;108(9):1335-40.

10. Steffens JC, Bourne MW, Sakuma H, et al. Quantification of collateral blood flow in coarctation of the aorta by velocity encoded cine magnetic resonance imaging. Circulation. 1994;90(2):937-43.

11. Muzzarelli S, Meadows AK, Ordovas KG, et al. Usefulness of cardiovascular magnetic resonance imaging to predict the need for intervention in patients with coarctation of the aorta. Am J Cardiol. 2012;109(6):861-5.

12. Darabian S, Zeb I, Rezaeian $P$, et al. Use of noninvasive imaging in the evaluation of coarctation of aorta. J Comput Assist Tomogr. 2013;37(1):75-8.

13. Kramer CM, Barkhausen J, Flamm SD, et al. Standardized cardiovascular magnetic resonance imaging (CMR) protocols, society for cardiovascular magnetic resonance: board of trustees task force on standardized protocols. J Cardiovasc Magn Reson. 2008;10(1):35.

14. Shaddy RE, Snider AR, Silverman NH, et al. Pulsed Doppler findings in patients with coarctation of the aorta. Circulation 1986;73:82-8.

15. Rao PS, Carey P. Doppler ultrasound in the prediction of pressure gradients across aortic coarctation. Am Heart J 1989;118:299-307.

\section{Correspondence to:}

Begoña Igual

Department of Cardiology

Consorci Hospital General Universitari de Valencia

Valencia, Spain

Email: bigual@icloud.com 